\title{
Excesso de peso em escolares: percepções e intercorrências na escola
}

\author{
Angela Maria Borges Miziara \\ Instituto de Psicologia da Universidade Federal de Uberlândia - MG \\ Celia Vectore \\ Instituto de Psicologia da Universidade Federal de Uberlândia - MG
}

\begin{abstract}
Resumo
Este artigo investiga como escolares obesos percebem a sua própria obesidade. Para tanto, após a realização da avaliação antropométrica de 622 crianças de idades entre seis anos e onze anos e onze meses, oriundas de uma escola pública do Interior de Minas Gerais foram identificadas 60 crianças obesas, e destas, 19 participaram da pesquisa. Os dados foram coletados em cinco oficinas psicopedagógicas e pela aplicação da Escala de Stress Infantil. Os resultados apontaram as dificuldades das famílias em lidar com a obesidade dos filhos, além de sentimentos contraditórios da criança quanto ao seu peso, manifestados ora por tristeza ora por raiva. Identificou-se também a presença de bullying no cotidiano dos participantes, especialmente na escola. Os indicadores de estresse apareceram em $21 \%$ das crianças. 0 estudo não permite generalizações, mas sugere a pertinência de trabalhos conjuntos, em equipe multiprofissional para o enfrentamento da obesidade.
\end{abstract}

Palavras-chave: obesidade; stress; testes psicológicos.

\section{Overweight children: perceptions and intercurrences at school}

\begin{abstract}
In this study we aim at investigating how obese school children react to their condition. Thus, an anthropomorphic evaluation of 622 children, six to eleven years of age, attending public schools in the interior of the State of Minas Gerais, was carried out. Sixty obese children were identified and nineteen of them participated in the research study. Data were collected in five psychopedagogical workshops where Stress Scale for Children was administered. Results indicate that families find it difficult to deal with their children's obesity. The researchers also found contradictory emotions, such as anger or sadness, demonstrated by the children in relation to their weight. There are also indications that overweight children were frequently bullied, in particular in school. Stress indicators suggest that twenty-one percent of the children surveyed suffered from stress. At present, generalizations cannot be made from the current study. However, it suggests the need for a multi-professional approach in dealing with childhood obesity.
\end{abstract}

Keywords: Obesity, stress, psychological testing.

\section{Exceso de peso en escolares: percepciones e complicaciones en la escola}

\section{Resumen}

Este artículo investiga como escolares obesos perciben su propia obesidad. Para ello, después de evaluación antropométrica de 622 niños de seis a once años y once meses de edad, estudiantes de una escuela pública del Interior de Minas Gerais, fueron identificados 60 niños obesos y de estos, 19 participaron de la investigación. Se recolectaron datos en cinco talleres psicopedagógicos y mediante aplicación de la Escala de Estrés Infantil. Los resultados señalaron dificultades de las familias para enfrentar la obesidad de los hijos, además de la presencia de sentimientos contradictorios en los niños acerca do su peso manifestados sea por tristeza sea por rabia. Se identificó también la presencia de bullying en el cotidiano de los participantes, especialmente en la escuela. Los indicadores de estrés aparecieron en el $21 \%$ de los niños. El estudio no permite generalizaciones, pero sugiere que el trabajo conjunto en equipo multiprofesional para el enfrentamiento de la obesidad es pertinente.

Palabras Clave: escolares; obesidad; estrés; pruebas psicológicas. 


\section{Introdução}

A adequada ingesta de nutrientes oriundos de uma alimentação saudável e balanceada tem sido um fator de manutenção da espécie humana no planeta; contudo, apesar de na atualidade haver maior disponibilidade de alimentos, o comportamento alimentar de uma considerável parcela da população não tem garantido de modo satisfatório, uma incorporação eficiente de nutrientes e, por outro lado, tem propiciado o aparecimento de um grande número de patologias diretamente ligadas à ingestão de alimentos altamente calóricos e não necessariamente nutritivos, com destaque para a obesidade e as doenças a ela atribuídas. Em acréscimo, as inadequações alimentares têm sido detectadas em idades cada vez mais tenras.

Nesse contexto, a Associação Dietética Americana (American Dietetic Association, 2008) elencou uma série de recomendações que enfatizam a importância de uma adequada nutrição para a promoção do desenvolvimento físico e cognitivo de crianças entre dois e onze anos, ressaltando o papel dos pais e cuidadores na formação de comportamentos alimentares saudáveis. Pesquisadores de Harvard criaram o Healthy Eating Plate, no entanto o programa já acumula críticas no que respeita aos tipos de alimento propostos para a composição do prato (Willett, \& Ludwig, 2011).

Várias medidas vêm sendo implementadas e avaliadas, entre elas o estudo de Thompson (2013), o qual identifica que as práticas alimentares de mães com sobrepeso ou obesas podem contribuir para o excesso de peso dos filhos e que práticas como o apoio ao aleitamento materno e a aprendizagem da relação entre alimentação, fome e saciedade se constituem em estratégias importantes para prevenir a transmissão intergeracional da obesidade. Tiffin e Arnoult (2011) realizaram uma investigação acerca do impacto da taxação de produtos gordurosos na saúde pública e concluíram que um imposto de gordura deve ser um dos componentes, dentre um conjunto de medidas a serem adotadas no combate à má alimentação, sendo necessário o envolvimento de diferentes segmentos da sociedade (comunidade, escola, família, indivíduo outros). Ludwig e cols. (2011) avaliaram que a própria mudança de um bairro com alto nível de pobreza para um de menor nível pode reduzir a incidência de obesidade e diabetes, embora tais mecanismos não estejam elucidados.

Compreender o intricado quebra-cabeça em que se constitui a construção do comportamento alimentar e suas disfunções tem incitado um grande número de estudos, que vão desde os que tentam identificar os aspectos bioquímicos ligados às estruturas cerebrais até os que constatam a existência de uma multiplicidade de fenômenos, presentes no ato de escolher e ingerir determinados alimentos. Tais aspectos se relacionam com variáveis individuais de natureza subjetiva e também variáveis culturais, as quais se vinculam e se entrelaçam na própria história do indivíduo.

Não obstante, estudos específicos que tenham originado propostas passíveis de serem aplicadas às crianças e seus cuidadores são escassos e seus resultados são incon- cludentes. Serdula, Alexander, Scanlon e Bowman (2001), ao fazerem uma revisão da literatura sobre a avaliação da ingestão alimentar entre pré-escolares, evidenciaram a importância de se dispor de instrumentos que, além de serem práticos, possam efetivamente avaliar com precisão a ingesta alimentar, permitindo a validade e a reprodutibilidade dos trabalhos relatados na literatura científica.

Essery, DiMarco, Rich, e Nichols (2008) avaliaram o impacto do fornecimento de informações periódicas, durante doze semanas, por meio de matérias escritas enviadas para mães de pré-escolares, referentes ao comportamento alimentar das crianças. Entre os resultados descritos, os autores observaram que as mães diminuíram a "pressão para comer" exercida sobre as crianças e concluíram que as práticas alimentares na infância são modificáveis.

O estudo brasileiro de Fiates, Amboni e Teixeira (2008), realizado com escolares de sete a dez anos de idade, mostrou a notável influência dos comerciais veiculados na televisão sobre as preferências alimentares. Segundo essas autoras, é necessário dispor de estratégias educacionais capazes de fomentar hábitos alimentares saudáveis.

Uma das consequências das inadequações alimentares é representada pela obesidade, uma "doença crônica, complexa, de etiologia multifatorial e resulta de balanço energético positivo. Seu desenvolvimento ocorre, na grande maioria dos casos, pela associação de fatores genéticos, ambientais e comportamentais" (Manual de Orientação da Sociedade Brasileira de Pediatria, 2008, p. 15). O documento informa a existência de mais de 400 genes responsáveis por codificar componentes ligados à regulação do peso corporal, todavia enfatiza a importância do ambiente na programação genética.

Além disso, estudos como o de Taveras, Rifas-Shiman, Oken, Gunderson e Gillman (2008), concluíram que bebês que dormem menos de doze horas diárias apresentam maior probabilidade de desenvolver obesidade. Acrescentam ainda que, se o sono for insuficiente e se estiver agregado com a exposição à televisão duas horas ou mais por dia, seu risco é ainda maior. Tal achado é corroborado pelo trabalho de Casturi e cols. (2011 citado por Preidt, 2011) referente à associação entre dormir menos de oito horas por noite e obesidade em crianças e adolescentes, causada pela diminuição do hormônio leptina, que é ligado à saciedade, e ao aumento da grelina, que estimula o apetite.

Dentre o caleidoscópio de possibilidades que envolvem a obesidade, suas causas e consequências, um fator que deve ser continuamente considerado são os aspectos emocionais da criança obesa. Campos, Sigulem, Moraes, Escrivão e Fisberg (1996, p.86) identificaram que "a imaturidade, a infantilização, a dependência, a passividade, a baixa autoestima, a falta de iniciativa e a dificuldade de adiar gratificações" são as características psicológicas mais citadas na literatura internacional relativa a crianças e adolescentes com excesso de peso. Por outro lado, para Cataneo, CarvaIho e Galindo (2005), os dados da literatura não são conclusivos, pois ser obeso não implica ter sentimentos negativos sobre seu corpo. 
A despeito da complexidade das variáveis que compõem o quadro da obesidade e o seu aumento avassalador na atualidade, tem-se que no Brasil, segundo a Pesquisa de Orçamentos Familiares, realizada em parceria pelo Instituto Brasileiro de Geografia e Estatística (IBGE, 2010) e pelo Ministério da Saúde, houve um aumento significativo do número de crianças com sobrepeso, principalmente entre aquelas com cinco a nove anos de idade. Em termos globais, o relatório "Estatísticas Mundiais da Saúde 2012" (WHO, 2012) informa que $12 \%$ da população mundial encontra-se obesa, alertando para o aumento das doenças não contagiosas relacionadas à obesidade, como diabetes, hipertensão arterial e doenças cardiovasculares.

Dutra de Oliveira (2005), importante pesquisador brasileiro da área de nutrição enfatiza que

(...) é preciso ensinar as pessoas a comerem nutricionalmente bem, e exigir políticas e programas que promovam a boa alimentação... É preciso ensinar como se alimentar nas escolas, pelo rádio, pela TV. Como pode existir nas escolas públicas cantinas vendendo tantos doces, refrigerantes e frituras aos alunos, e não leite, frutas ou sucos naturais?
Destarte, não é tarefa fácil responder à pergunta acima, e todo esforço no sentido de contribuir para a mudança do comportamento alimentar deletério de nossas crianças é bem-vindo. A partir dessas considerações, este trabalho objetivou conhecer como os escolares percebem a sua própria obesidade, seus sentimentos em relação ao próprio corpo e as possíveis intercorrências no ambiente escolar (rejeição dos colegas e outros), além de identificar a presença de estresse nos participantes.

\section{Método}

\section{Participantes}

Participaram deste estudo 19 crianças (oito meninas e onze meninos) identificadas como obesas, com idades entre sete e onze anos, sendo a média de oito anos e quatro meses, matriculadas entre o primeiro e o quarto anos do Ensino Fundamental de uma escola pública da cidade de Uberlândia, Minas Gerais. A tabela 1 mostra a configuração da amostra de participantes, a partir da avaliação antropométrica.

Tabela 1. Avaliação antropométrica das crianças participantes do estudo

\begin{tabular}{|c|c|c|c|c|c|c|c|c|}
\hline \multirow{2}{*}{ Criança } & \multirow{2}{*}{ Sexo } & \multirow{2}{*}{$\begin{array}{l}\text { Idade } \\
\text { (anos) }\end{array}$} & \multirow{2}{*}{$\begin{array}{l}\text { Peso } \\
(\mathrm{Kg})\end{array}$} & \multirow{2}{*}{$\begin{array}{c}\text { Altura } \\
\text { (cm) }\end{array}$} & \multicolumn{4}{|c|}{ Classificação } \\
\hline & & & & & P/A & $\mathrm{P} / \mathrm{I}$ & $A / I$ & Obesidade \\
\hline $1^{a}$ & Fem & 7 & 39,1 & 137,0 & $\mathrm{P}>95$ & $\mathrm{P}>95$ & $\mathrm{P}>95$ & Obesa \\
\hline $2^{a}$ & Fem & 9 & 47,7 & 144,0 & $\mathrm{P}>95$ & $\mathrm{P}>95$ & $P>95$ & Obesa \\
\hline $3^{a}$ & Masc & 9 & 47,6 & 141,0 & $\mathrm{P}>95$ & $\mathrm{P}>95$ & $\mathrm{P}>95$ & Obeso \\
\hline $4^{a}$ & Masc & 8 & 47,2 & 137,5 & $P>95$ & $P>95$ & P95 & Obeso \\
\hline $5^{a}$ & Fem & 10 & 52,8 & 149,0 & $P>95$ & $P>95$ & P95 & Obesa \\
\hline $6^{a}$ & Fem & 10 & 51,2 & 147,0 & $\mathrm{P}>95$ & $\mathrm{P}>95$ & P95 & Obesa \\
\hline $7^{a}$ & Mas & 11 & 84,5 & 158,0 & $\mathrm{P}>95$ & $\mathrm{P}>95$ & $P>95$ & Obeso \\
\hline $8^{a}$ & Masc & 9 & 49,3 & 149,5 & $\mathrm{P}>95$ & $\mathrm{P}>95$ & $\mathrm{P}>95$ & Obeso \\
\hline $9^{a}$ & Masc & 9 & 45,9 & 141,0 & $P>95$ & $\mathrm{P}>95$ & P75-P90 & Obeso \\
\hline $10^{a}$ & Fem & 7 & 33,0 & 127,0 & $P>95$ & $P>95$ & P75-P90 & Obesa \\
\hline $11^{a}$ & Fem & 7 & 41,1 & 132,0 & $\mathrm{P}>95$ & $P>95$ & $\mathrm{P}>75$ & Obesa \\
\hline $12^{\mathrm{a}}$ & Masc & 7 & 48,2 & 146,5 & $\mathrm{P}>95$ & $\mathrm{P}>95$ & $\mathrm{P}>95$ & Obeso \\
\hline $13^{a}$ & Masc & 10 & 48,2 & 146,5 & $P>95$ & $P>95$ & $P>95$ & Obeso \\
\hline $14^{\mathrm{a}}$ & Fem & 8 & 53,4 & 138,0 & $P>95$ & $P>95$ & P95 & Obesa \\
\hline $15^{\mathrm{a}}$ & Masc & 9 & 53,5 & 145,0 & $P>95$ & $\mathrm{P}>95$ & $P>95$ & Obeso \\
\hline $16^{a}$ & Masc & 10 & 76,5 & 151,0 & $P>95$ & $P>95$ & $P>95$ & Obeso \\
\hline $17^{a}$ & Masc & 10 & 50,7 & 150,0 & P95 & $P>95$ & P95 & Obeso \\
\hline $18^{a}$ & Masc & 11 & 57,3 & 157,0 & P95 & $P>95$ & P95 & Obeso \\
\hline $19^{a}$ & Fem & 11 & 51,4 & 152,0 & P90-P95 & P90-P95 & P75-P90 & Obeso \\
\hline
\end{tabular}




\section{Instrumentos}

Para a avaliação antropométrica foram utilizados uma balança digital e um estadiômetro. A realização das oficinas envolveu o uso de fantoches, bonecos e outros recursos lúdicos. A sintomatologia indicativa de estresse foi avaliada pela Escala de Stress Infantil- ESI (Lipp, \& Lucarelli, 2005), que contempla em seus 35 itens quatro fatores relativos a reações físicas, reações psicológicas, reações psicológicas com componente depressivo e reações psicofisiológicas.

\section{Procedimentos}

Das 840 crianças matriculadas na escola, 622 escolares participaram da avaliação antropométrica feita pela nutricionista, colaboradora do estudo. A avaliação incluiu crianças de ambos os sexos, estudantes do $1^{\circ}$ ao $4^{a}$ ano do Ensino Fundamental, com idades de seis anos a onze anos e onze meses. Para as crianças de idade entre seis e oito anos e onze meses de idade foi utilizado o padrão antropométrico com indicadores Peso/Altura, Altura/ldade e Peso/Idade do Centers for Disease Control and Prevention/ National Center for Health Statistics ([CDC/ NCHS], 2000). Para as crianças entre nove anos e onze anos e onze meses de idade foi calculado o IMC [P $\left.(\mathrm{Kg}) / \mathrm{A}^{2}\left(\mathrm{~m}^{2}\right)\right]$, indicado pelo National Health and Nutrition Examination Survey ([NHANES], 2001).

As crianças foram classificadas como eutróficas, pré-obesas ou obesas de acordo com os indicadores acima citados. Das sessenta crianças obesas detectadas pela avaliação empreendida, foram escolhidas aleatoriamente trinta e duas para participar do estudo, o que correspondeu a aproximadamente metade da população avaliada. Isto foi tido pertinente aos propósitos da investigação e às limitações inerentes à pesquisa, como tempo para a conclusão, recursos e outras. Das crianças escolhidas, vinte e três receberam o consentimento dos pais, que foram convidados para uma reunião específica para a explicação do estudo. Quatro crianças não compareceram a todas as atividades previstas na pesquisa, o que reduziu a amostra inicial para 19 participantes.

Com os participantes foram realizadas, na própria escola, cinco oficinas, com duração de duas horas e trinta minutos cada. Tais oficinas, de natureza lúdica, foram organizadas com o intuito de promover a expressão e a reflexão dos participantes sobre temas relativos à própria obesidade. As crianças foram divididas em dois grupos, de acordo com o horário das aulas, de modo a se terem dez crianças nas atividades pela manhã e nove à tarde. Sinteticamente, na primeira oficina foi estabelecido o rapport entre a pesquisadora e as crianças, com explicitação dos objetivos do estudo e das normas de conduta durante os encontros; na segunda e terceira oficinas foram utilizadas dinâmicas com brincadeiras e outros recursos lúdicos; na quarta oficina foi aplicada coletivamente a Escala de Stress Infantil - ESI (Lipp, \& Lucarelli, 2005), e na quinta oficina foi realizada uma reflexão sobre todas as atividades desenvolvidas durante as oficinas.

Os encontros foram áudio gravados e transcritos para posterior análise e identificação das categorias. Este estudo teve a aprovação do Comitê de Ética em Pesquisa da Universidade Federal de Uberlândia (Protocolo $n^{\circ} 373 / 05$ ) e todos os participantes da amostra assinaram o Termo de Consentimento Livre e Esclarecido.

\section{Resultados e discussão}

As 622 crianças tiveram suas alturas e pesos aferidos para o cálculo dos índices antropométricos (peso/altura, altura/idade e peso/idade). A partir desses dados, constatou-se um total de 60 crianças obesas, como se vê na tabela 2.

Tabela 2. Prevalência da obesidade conforme idade, em alunos de uma escola pública.

\begin{tabular}{lcc}
\hline Idade & Total alunos & $\%$ obesos \\
\hline 6 anos & 6 & 10,0 \\
7 anos & 15 & 25,0 \\
8 anos & 12 & 20,0 \\
9 anos & 16 & 26,7 \\
10 anos & 6 & 10,0 \\
11 anos & 5 & 8,3 \\
\hline Total & 60 & 100,0
\end{tabular}

Isto significa que, dos 622 alunos avaliados, cerca de $10 \%$ estavam obesos. Foi encontrada uma maior concentração de obesos em turmas do $2^{\circ}$ e $4^{\circ}$ anos. Observou-se ainda que o percentual de obesos do período da tarde é maior que o do período da manhã (6\% - manhã e $12 \%$ tarde), dado que deve ser mais bem investigado, pois não foi possível identificar nenhum fator que pudesse explicá-lo adequadamente. Além disso, das 60 crianças obesas, 35 eram meninos e 25 , meninas, dado corroborado pelo IBGE (2010), que apontou um percentual maior de obesidade entre os meninos brasileiros.

No encontro inicial, realizado com os pais na escola, foram elencadas algumas dificuldades enfrentadas em relação à alimentação dos filhos, como: desconhecimento de uma alimentação nutricionalmente adequada; grande consumo de comidas calóricas e de baixo valor nutricional, indicador corroborado pelo estudo do IBGE (2011); dificuldades econômicas, não permitindo gastos com academias, por exemplo; atitudes diferenciadas de pais quanto à alimentação dos filhos; e finalmente, baixo envolvimento dos professores quando as crianças são alvo de críticas maldosas pelos pares. Segundo eles, os professores "não veem ou não querem ver o que está acontecendo". 
É interessante constatar que vários dos aspectos citados pelos pais foram confirmados nos trabalhos com as crianças, o que significa que a questão da alimentação envolve uma multiplicidade de variáveis, as quais envolvem atitudes da família, da escola - que disponibiliza refeições calóricas, sem um rígido controle do que é ofertado -, e os professores, que por não disporem de recursos suficientes para lidar com situações de conflito entre os alunos acabam por gerar e manter um círculo vicioso, no qual a indiferença se destaca. Gonçalves, Piovesan, Link, Prestes e Lisboa (2005) sugerem que "ações educativas (dramatização, dilemas morais e dinâmicas de grupo) que visam a favorecer a emergência de interação social construtiva" (p.635) podem contribuir com a formação de professores, possibilitando um maior e melhor enfrentamento dessas questões.

Em relação às oficinas realizadas com as crianças, observou-se que no início algumas crianças mostravam certa timidez, mas ao longo dos trabalhos houve um maior envolvimento com o grupo, o que permitiu relatos de suas ansiedades e tristezas devido ao excesso de peso. As oficinas foram transcritas e analisadas em seu conteúdo (Bardin, 2008), o que resultou na identificação de duas categorias de análise dos relatos verbais: 1 - Aceitação de si e pelos pares e sentimentos frequentes, e 2- Rotinas: preferências alimentares e sedentarismo. Para a apresentação dos dados, além das categorias indicadas, far-se-ão referência aos resultados obtidos na Escala de Stress Infantil-ESI (Lipp, \& Lucarelli, 2005).

\section{frequentes}

\section{1) Aceitação de si e pelos pares e sentimentos}

Quanto à aceitação de si, a ampla maioria dos participantes disse não ter problemas com o excesso de peso, mas alguns participantes expressam descontentamento. As frases abaixo ilustram essa situação, sendo que o primeiro exemplo ilustra ausência de descontentamento e o segundo exemplo, o descontentamento:

"(...) sou bem tranquilo, sem problema, me sinto muito bem todos os dias!” (criança 4).

"(...) eu fico muito triste com o meu corpo, não queria ser gorda!" (criança 11).

No que concerne à aceitação dos pares, os sentimentos mais frequentes são a tristeza por estar acima do peso e a raiva por ser alvo de chacotas e apelidos pejorativos. Destaca-se ainda o desejo de agredir os provocadores, principalmente entre os meninos. As meninas se portam de modo mais contido, normalmente choram e reclamam para os professores e genitores. Os excertos abaixo identificam tais aspectos:

"(...) eu me sinto triste, quando eles me chamam de gordo (...) eu queria brincar de bola com um colega e ele não deixou e ainda me xingou de gordo e eu furei a bola dele!" (criança 3).
“(...) muitos me xingam de Bolinha, o meu pai me chama de Bolão, uma vez minha irmã me xingou e eu quebrei os dois dentes dela!" (criança 8).

“(...) eu fico muito magoada, triste e até ameaçada quando me chamam de hipopótamo, baleia e de quatro olhos" (usa óculos) (criança 5).

"(...) quando os meninos da escola mexem comigo eu fico muito triste, às vezes eu choro. Fico quieta na sala e não conto para a professora e nem para o meu pai, só para a minha mãe" (criança 10).

“(...) não fico triste por ser gordo. Fico com muita raiva quando os meninos mexem comigo, tenho vontade de partir pra cima e bater em todo mundo" (criança 12).

Além disso, manifestações de bullying foram identificadas durante as duas primeiras oficinas, nos dois grupos participantes. Lopes Neto (2005, p.165) argumenta que bullying "compreende todas as atitudes agressivas, intencionais e repetidas, que ocorrem sem motivação evidente, adotadas por um ou mais estudante contra outro(s)". No caso desta pesquisa o bulliying se deu pela colocação de apelidos pejorativos entre as próprias crianças obesas. Cumpre reconhecer que, diante dos sentimentos negativos engendrados no cotidiano da escola é preciso desenvolver ações que, além de coibir tais manifestações, provoquem reflexões e mudanças de comportamento. Freire e Aires (2012) argumentam que a atuação do psicólogo na escola pode promover a melhoria das relações sociais dentro desse contexto. Além disso, no caso específico do bullying, Silva e Rosa (2013) e Sánchez (2013) mostram a pertinência do espaço escolar para o estudo e as intervenções em situações de constrangimento inerentes a tal fenômeno. Em se tratando do excesso de peso no contexto escolar, é preciso haver o atendimento de uma equipe multiprofissional composta por psicólogo, educador físico e nutricionista. Neste sentido, a escola é um local privilegiado para uma abordagem efetiva de desse problema.

Embora alguns participantes digam não gostar do próprio corpo e expressem o desejo de emagrecer, são praticamente inexistentes ações voltadas ao enfrentamento do excesso de peso. Entre os participantes, apenas uma menina menciona fazer caminhadas e cuidar da alimentação, no que ela afirma contar com o apoio da mãe, conforme se observa nos relatos a seguir:

“(...) Não gosto do meu corpo, me sinto feio por ser gordo" (criança18).

“(...) Eu sei que estou acima do peso, mas não fico triste; faço caminhada, como muita fruta e verduras e ainda ando de bicicleta. Minha mãe me ajuda muito" (criança 19).

O contexto familiar é uma das variáveis de maior importância para o enfrentamento do excesso de peso. A 
revisão de estudos acerca de programas de intervenção quanto à obesidade infantil empreendida por Epstein, Wing, Koeske e Valoski (1987) mostrou que os mais efetivos são os que envolvem a família. Nesse sentido, sensibilizar os familiares e torná-los corresponsáveis por tal enfrentamento deve ser um dos fatores a serem considerados quando se vislumbram alternativas de alteração comportamental.

\section{sedentarismo \\ 2. Rotinas: preferências alimentares e \\ Os participantes relataram suas preferências alimen-} tares e também tentaram explicar a causa do excesso de peso. Entre os alimentos preferidos se encontram com frequência cachorro-quente, coxinha, bolachas de chocolate, chocolate, macarrão instantâneo, pizza, lasanha e doces, conforme se depreende das falas abaixo:

\section{(...) Eu gosto muito de tomate e pizza (criança 4). \\ (...) Gosto de comer pão, bolacha, arroz, feijão, salada (criança 11).}

\section{(...) O que eu mais gosto de comer é chocolate (criança 9).}

Ingerir cotidianamente alimentos calóricos e de baixo valor nutricional compõe a rotina dos participantes. Apenas uma das crianças mencionou alimentar-se adequadamente, pelo menos em se tratando de qualidade nutricional (arroz, feijão, verduras, etc.); não foi possível avaliar quanti e qualitativamente a ingesta alimentar. Svedberg (2011) mostra que estar acima do peso se constitui na principal forma de desnutrição na atualidade, não só nos países desenvolvidos, mas também em quase todos os países em desenvolvimento.

Em acréscimo, atrelado à ingesta alimentar inadequada, os participantes demonstram pouco envolvimento com atividades físicas como jogos e brincadeiras ao ar livre ou na escola; em geral, assistem televisão por um período de duas a cinco horas diariamente, conforme pode ser observado na tabela 3 e no comentário de um dos participantes.

Fico assistindo televisão até às quatro horas da manhã e não tenho hora para levantar, porque estudo de tarde (criança 13).

Tabela 3. Tempo em que as crianças ficam em frente à televisão.

\begin{tabular}{lll}
\hline Tempo em horas & $\mathrm{N}$ & $\%$ \\
\hline Menos $2 \mathrm{~h}$ & 7 & 36,8 \\
$2-5 \mathrm{~h}$ & 8 & 42,1 \\
$5-10 \mathrm{~h}$ & 4 & 21,1 \\
\hline
\end{tabular}

As crianças ainda relatam ser mais lentas e menos ágeis, especialmente nas aulas de Educação Física. Em relação a esse dado, um dos participantes se exprime:

Na hora de jogar bola os meninos me rejeitam por eu ser mais lento, sempre fico para trás (criança 17).

A ausência de atividades físicas na rotina da maioria dos participantes mostra a importância do professor de Educação Física, que deve criar no âmbito escolar estratégias para o envolvimento dessas crianças que contribuam para um maior gasto energético e promovam a autoestima e a socialização entre elas. Guedes e Guedes (1998) ressaltam a posição privilegiada ocupada por esse profissional na promoção e execução de tais iniciativas.

A alta ingestão calórica e o sedentarismo resultam em um balanço energético positivo, onde se dispõe de mais energia que a necessária para o adequado funcionamento do organismo, dando origem ao excesso de peso. Tal dado demonstra a necessidade de orientação familiar para o estabelecimento e o controle de rotinas mais saudáveis para as crianças. Vários participantes relataram que ficam um grande período sem supervisão familiar, devido ao trabalho dos pais:

(...) Fico em casa sozinho e como o que quero, mas a minha mãe deixa o meu prato pronto, antes de sair para o trabalho (criança 7).

Outro dado digno de nota é o descompasso de comportamentos existentes no contexto familiar no tocante à alimentação das crianças. Comidas não disponibilizadas por um dos genitores, normalmente doces, são ofertadas como forma de gratificação pelo outro genitor ou um parente, conforme se depreende dos excertos a seguir:

(...) quando meu pai recebe, ele compra muitos doces pra nós! (criança 11).

(...) tento controlar a alimentação dela, mas o avô sempre oferece alimentos calóricos e guloseimas! (criança 14).

Oferecer às crianças guloseimas calóricas, normalmente adocicadas e gordurosas, é uma estratégia culturalmente aceita no nosso contexto. O Manual de Orientação elaborado pela Sociedade Brasileira de Pediatria (2008) alerta que "mães que trabalham fora, por exemplo, costumam 'compensar' sua ausência levando guloseimas para os filhos, como um modo de 'adocicar' o sentimento ansioso e de 'acalmar' o desconsolo que ele causa" (p. 20), evidenciando uma associação precoce e inadequada entre a comida e modos de se lidar com situações difíceis ou complexas.

Sobre isso cabe uma pergunta: afinal, qual a percepção das crianças sobre seu excesso de peso? Responder a essa questão é um tarefa árdua, já que a obesidade tem etiologia multifatorial; contudo chama a atenção o fato de dois participantes mencionarem que comem quando 
Tabela 4. Prevalência de reações ao estresse nos participantes, segundo o gênero.

\begin{tabular}{|c|c|c|c|c|c|c|c|c|c|c|}
\hline & \multirow{2}{*}{$\mathrm{N}$} & \multirow{2}{*}{$\%$} & \multicolumn{4}{|c|}{ Indicadores de Estresse } & \multicolumn{2}{|c|}{$\begin{array}{l}\text { Sem Indicadores de } \\
\text { Estresse }\end{array}$} & \multirow[t]{2}{*}{$\Sigma$} & \multirow[t]{2}{*}{$\Sigma \%$} \\
\hline & & & Fís. & Psic. & $\begin{array}{l}\text { Psic. comp. } \\
\text { depres. }\end{array}$ & Psicof. & $\mathrm{N}^{\circ}$ & $\%$ & & \\
\hline Meninos & 3 & 27,3 & & & 3 & & 8 & 72,7 & 11 & 100 \\
\hline Meninas & 1 & 12,5 & & 1 & & & 7 & 87,5 & 8 & 100 \\
\hline
\end{tabular}

estão tristes, ou nos dizeres da criança 8: "a gente engorda por causa da ansiedade, come, come, come e não consegue parar!". Luiz, Gorayeb, Liberatore Júnior e Domingos (2005) referem-se à existência da relação entre a obesidade infantil e aspectos psicológicos como depressão, ansiedade e déficits de competência social, alertando para a necessidade de uma investigação mais acurada acerca do fenômeno.

3. Resultados da Escala de Stress Infantil - ESI

No que concerne à aplicação da Escala de Stress Infantil - ESI (Lipp \& Lucarelli, 2005), a tabela 4 trata da caracterização dos participantes quanto ao gênero e à presença ou não de reações ao estresse.

Os resultados da Escala de Stress Infantil - ESI permitem concluir que quatro participantes apresentaram reações indicativas de estresse - três meninos e uma menina. Assim, quando se considera apenas o gênero, tem-se que, proporcionalmente, de cada dez meninos, três apresentaram reações ao estresse, notadamente "Reações Psicológicas com Componentes Depressivos", conforme avalia o instrumento. As reações psicológicas com componente depressivo mais frequentes foram: "não tenho vontade de fazer as coisas" e "sinto que tenho pouca energia para fazer as coisas". Entre as meninas a proporção foi de cerca de uma a cada dez.

Embora não se possa fazer nenhum tipo de generalização, dentre os participantes deste estudo os meninos parecem estar mais sujeitos às reações de estresse. Tal dado não é corroborado por trabalhos anteriores, como o de Lipp (2004) e Rodrigues (2006), que encontraram a prevalência de estresse em meninas. Assim, novas pesquisas, com amostras ampliadas e significativas, são necessárias para se saber se na população infantil com excesso de peso os meninos estão realmente mais sujeitos a apresentar comportamentos sugestivos de estresse. A despeito da necessidade de um diagnóstico mais acurado e conclusivo, esse dado é preocupante, independentemente do gênero, devido aos efeitos deletérios causados pelo estresse e pela sua potencialidade em comprometer a qualidade de vida já na infância.

Outro dado que merece destaque se refere ao fato de a grande maioria dos participantes não fazer nenhum tipo de tratamento ou intervenção para reduzir o peso. Embora tenha havido menção a consultas com nutricionistas e médicos, as orientações recebidas pelos profissionais não foram agregadas à rotina de modo decisivo ou contínuo. Apenas uma criança mencionou fazer tratamento para o excesso de peso, por apresentar disfunção na tireoide (criança 11). Fett, Fett, Padovan e Marchini (2009) argumentam que "os hormônios da tireoide são potentes moduladores da termogênese e podem, potencialmente, contribuir para o desenvolvimento da obesidade" (p. 10).

Os dados indicam um aparente descaso com o problema do excesso de peso nos contextos dos quais essas crianças participam. Além disso, revelam a pouca adesão aos tratamentos nas raras vezes em que estes são disponibilizados. Por isso várias estratégias oriundas das diferentes áreas que investigam tal fenômeno devem ser veiculadas e agregadas, no sentido de conscientizar as pessoas quanto aos malefícios da obesidade. É ingênuo acreditar que apenas intervenções isoladas, priorizando ora aspectos nutricionais ora comportamentais, possam efetivamente dar conta da complexidade que envolve o crescente ganho de peso na população.

\section{Considerações finais}

Conhecer como as crianças reagem à sua própria obesidade e às suas possíveis intercorrências no ambiente escolar foi o objetivo perseguido neste estudo. É importante destacar que, embora a ênfase tenha recaído sobre as variáveis comportamentais, a ampla avaliação nutricional realizada nas crianças permitiu identificar que cerca de $10 \%$ delas apresentaram excesso de peso, o que por si só já lança luz sobre a necessidade de políticas públicas efetivas para deter o avanço de tal epidemia.

Em acréscimo, acredita-se que envolver as famílias e profissionais da educação em programas de conscientização sobre a importância de uma alimentação saudável que não contemplem apenas informações sobre os alimentos de um ponto de vista meramente nutricional, mas abarquem também as variáveis emocionais presentes no ato de comer, é uma alternativa que merece estar presente nos contextos educacionais. Neste sentido, a escola pode ser uma inte- 
ressante porta de acesso a iniciativas voltadas à formação dos professores, à educação ou reeducação nutricional, ao incremento de atividades físicas e a outros aspectos da educação. Vale destacar a pertinência de que tais iniciativas sejam coordenadas por uma equipe multiprofissional.

Por fim, é desejável que estudos em diferentes áreas que tenham como foco o excesso de peso das crianças brasileiras redundem em iniciativas que efetivamente minimizem os riscos a que elas se encontram expostas. Ações voltadas à educação nutricional já nos primeiros anos de vida, além do combate às propagandas de alimentos nutricionalmente inadequados, acrescidas de programas para a sensibilização dos malefícios do excesso de peso, certamente contribuirão para termos uma geração saudável em todos os sentidos e apta à vivência harmônica numa sociedade plural, o que se constitui num dos grandes desafios da contemporaneidade.

\section{Referências}

American Dietetic Association. (2008). Position of the American Dietetic Association: Nutrition Guidance for Healthy Children Ages 2 to 11 Years. Journal of the American Dietetic Association, 108(6), 1038-1047.

Campos, A. L. R., Sigulem, D. M., Moraes, D.E.B., Escrivão, A. M.S., \& Fisberg, M. (1996). Quociente de inteligência de crianças e adolescentes obesos através da Escala Wechsler. Revista Saúde Pública, 30(1), 85 -90.

Bardin, L. (2008). Análise de conteúdo. Lisboa: Edições 70.

Cataneo, C., Carvalho, A. M. P., \& Galindo, E. M. C. (2005). Obesidade e Aspectos Psicológicos: Maturidade Emocional, Auto-conceito, Locus de Controle e Ansiedade. Psicologia: Reflexão e Crítica, 18(1), 39-46.

Centers for Disease Control and Prevention and National Center for Health Statistics (2000). Recuperado: 25 out. 2011. Disponível: http:// www.cdc.gov/nchs/about/major/nhanes/gcrevision.htm

Epstein L., Wing, R. R., Koeske, R., \& Valoski, A. (1987). Long-term effects of family based treatment of childhood obesity. Journal of Consulting and Clinical Psychology, 55, 91-95.

Essery, E. V., DiMarco, N. M., Rich, S.S., \& Nichols, D. L. (2008). Mothers of Preschoolers Report Using Less Pressure in Child Feeding Situations Following a Newsletter Intervention. Journal of Nutrition Education \& Behavior, 40(2), 110-116.

Fett, C. A.; Fett, W. C. R.; Padovan, G. J. \& Marchini, J. S. (2009). Associação do TSH, do gasto energético de repouso e do zinco na composição corporal de mulheres com sobrepeso/obesas. Arquivos de Ciências da Saúde, 16(1), 9-14.

Fiates, G. M. R., Amboni, R. D. M. C., \& Teixeira, E. (2008). Comportamento consumidor, hábitos alimentares e consumo de televisão por escolares de Florianópolis. Revista de Nutrição, 21(1), 105-114.

Freire, A. N. \& Aires, J. S. (2012). A contribuição da Psicologia escolar na prevenção e enfrentamento do Bullying. Psicologia Escolar e Educacional, 16(1), 55-60.

Gonçalves, M. A. S., Piovesan, O. M., Link, A., Prestes, L. F., \& Lisboa, J. G. (2005). Violência na Escola, Práticas Educativas e Formação do Professor. Cadernos de Pesquisa, 35(126), 635-658.

Guedes, D. P. \& Guedes, J. E. R. P.(1998). Prevalência de sobrepeso e obesidade em crianças e adolescentes do município de Londrina (PR), Brasil. Motriz, 4(1), 18-25.

IBGE (2010). POF 20082009 - Antropometria e estado nutricional de crianças, adolescentes e adultos no Brasil. Brasília: Autor.

IBGE (2011). Avaliação nutricional da disponibilidade domiciliar de alimentos no Brasil 2008-2009. Brasília: Autor.

Lipp, M. E. N. (2004). Crianças estressadas: causas, sintomas e soluções. São Paulo: Papirus.

Lipp, M. E.N., \& Lucarelli, M. D.M. (2005). ESI - Escala de stress infantil. São Paulo: Casa do Psicólogo.

Lopes Neto, A. A. (2005). Bullying - comportamento agressivo entre estudantes. Jornal de Pediatria, 81(5), 164- S172.

Ludwig, J., Sanbonmatsu, L., Gennetian,L. Adam, E., Duncan, G.J., Katz, L.F., Kessler, R.C, Kling, J.R., Lindau, S. T., Whitaker, R.C., \& McDade, T.W. (2011). Neighborhoods, Obesity, and Diabetes-A Randomized Social Experiment. The New England Journal of Medicine, 365(16), 1509-1519.

Luiz, A. M. A.G., Gorayeb, R., Liberatore Júnior, R. D. R., \& Domingos, N. A. M. (2005). Depressão, ansiedade e competência social em crianças obesas. Estudos de Psicologia, 10(1), 35-39.

National Health and Nutrition Examination Survey. (2001). National Health and Nutrition Examination Survey 2001-2002. Recuperado: 25 out. 2011. Disponível: http://www.cdc.gov/nchs/about/major/ nhanes/NHANES99_00.htm

Preidt, R. (2011). Sleepy Teens More Prone to Weight Gain: Study. Recuperado: 25 out. 2011. Disponível: http://www.realage.com/ shape-up-slim-down/

Oliveira, J. E. D. (2005). Nutrição deve ser pauta de saúde pública. Revista eletrônica Consciência da SBPC. Recuperado: 21 out. 2011. Disponível: http://www.com ciencia.br/ entrevistas/2005/09/ entrevista2.htm

Rodrigues, I. (2006). Incidência de estresse infantil em uma escola da rede privada de Teresina. Anais do IV Encontro de Pesquisa em Educação da Universidade Federal Piauí. Recuperado: 25 
out. 2012. Disponível: http://www.ufpi.edu.br/subsiteFiles/p pged/ arquivos/files/eventos/2006.gt15/GT15_2006_06.PDF

Sánchez, R. Y. (2013). Intervención educativa para resolver un caso de acoso escolar. Psicologia Escolar e Educacional, 17(2), 339354.

Serdula, M. K., Alexander, M. P., Scanlon, K.S., \& Bowman, B. A. (2001). What are prescholol children eating? A Review of Dietary Assessment. Annual Review of

Nutrition, 21, (pp. 475-498)

Silva, E. N., \& Rosa, E. C. S. (2013). Professores sabem o que é bullying? Um tema para a formação docente. Psicologia Escolar e Educacional, 17(2), 329-338.

Sociedade Brasileira de Pediatria (2008). Obesidade na infância e adolescência - Manual de Orientação. São Paulo: Autor.

Svedberg, P. (2011). How Many People Are Malnourished? Annual Review of Nutrition, 31, (pp. 263-283).
Taveras, E.M., Rifas-Shiman, S.L., Oken. E., Gunderson, E.P., \& Gillman, M.W. (2008). Short sleep duration in infancy and risk of childhood overweight. Archives of Pediatric and Adolescent Medicine,162(4), 305-311.

Thompson, A. L. (2013). Intergenerational impact of maternal obesity and postnatal feeding practices on pediatric obesity. Nutrition Reviews, 71(Suppl. 1), 555-561.

Tiffin, R., \& Arnoult, M. (2011). The public health impacts of a fat tax. European Journal of Clinical Nutrition,65, 427-433.

Willett, W. C., \& Ludwig, D. S. (2011). The 2010 Dietary Guidelines The Best Recipe for Health? The New England Journal Medicine, 365(17), 1563-1565.

World Health Organization. (2012). World Health statistcs. Recuperado: 05 jun. 2012. Disponível: http://www.who.int/gho/ publications

\section{Sobre as autoras}

Angela Maria Borges Miziara (amiziara@gmail.com)

Psicóloga e especialista em Psicopedagogia.

Celia Vectore (vectore@ufu.br)

Professora associada 4- Instituto de Psicologia da Universidade Federal de Uberlândia, doutora em Psicologia.

As autoras agradecem a nutricionista Ana Paula Borges Miziara, Mestre em Nutrição Humana/USP-SP e Docente da Pontifícia Universidade Católica de Goiás, que realizou toda a avaliação antropométrica dos escolares para a constituição da amostra dos participantes. 